\title{
Facemasks are Not Effective for Preventing Transmission of the Coronavirus
}

\author{
Colin A. Ross* \\ Institute for Psychological Trauma, 1701 Gateway, Suite 349, Richardson, TX 75080, USA
}

${ }^{\star}$ Corresponding author: Colin A. Ross, Institute for Psychological Trauma, 1701 Gateway, Suite 349, Richardson, TX 75080, USA

Received: August 06, 2020; Accepted: August 12, 2020; Published: August 18, 2020

On February 29, 2020, Jerome Adams, the Surgeon General of the United States, tweeted that, "They [facemasks] are NOT effective in preventing general public from catching coronavirus." Within a few months, he and the Centers for Disease Control and Prevention (CDC) were stating that facemasks are effective for preventing transmission of viral illnesses in the community, and within a short time, governments in the United States and elsewhere were mandating the wearing of facemasks in public. The explanation for this reversal in policy focused on asymptomatic carriers in public. However, the evidence from Randomized Controlled Trials (RTCs) indicates that surgical facemasks have no effect on the transmission of viral illnesses in the community. Based on their physical characteristics, one would not expect surgical facemasks to reduce viral transmission by asymptomatic carriers. The size of a coronavirus is about 0.1 microns and the pore size of surgical masks is in the range of 50-100 microns: the pores are 500-1000 times the size of the virus. Aerosol droplets are about 3 microns in diameter, so surgical masks would not be expected to block them either.

A common rationale for wearing masks in public is that their purpose is not to protect you from others, but to protect others from you if you are an asymptomatic carrier: this is illogical. How can a mask protect someone else from you if it does not protect you from someone else? Also, asymptomatic carriers are, by definition, not coughing or sneezing in public, except very infrequently. Normal breathing and speaking emits primarily aerosols not droplets; droplets are the main concern for transmission of the coronavirus in public. Infectious symptomatic carriers who are emitting virus-laden droplets should be in quarantine, therefore it is not necessary to mandate facemasks to protect the public from them.

Four meta-analyses published to date report that no Randomized Controlled Trial (RCT) has ever shown a significant difference in viral transmission rate with and without facemasks in the general public. Brainard, Jones, Lake, Hopper and Hunter [1] reviewed 3 RCTs and found no difference in any of them; Cowling, Zhou, Ip, Leung, and Aiello [2] reviewed 4 RCTs and found no difference in any of them; Xiao, Shiu, Gao, Wong, Fong, Ryu and Cowling [3] reviewed 10 RCTs and found no difference in any of them; and Aggarwal, Dwarakananthan, Gautam and Ray [4] reviewed 9 RCTs and found no difference in any of them. These meta-analyses by four different groups from around the world have not found a single RCT that demonstrates a protective effect of facemasks for viral transmission in the general public. In discussing the Xiao et al. [3] Meta-analysis, Greenlagh, Schmid, Czypionka, Bassler and Gruer [5] said that, “...the authors conclude that there was no significant reduction in influenza transmission with the use of facemasks." The situation, then, is not that there is a lack of evidence: there is replicated, controlled evidence that facemasks do not reduce viral transmission in the community.

For the sake of discussion, what if we made an assumption that facemasks reduce the rate of viral transmission in the community by $5 \%$ ? For this discussion, we will bear in mind that there is no evidence that facemasks reduce transmission by even this much. Further, let's assume that the infection rate in the community is $5 \%$ and the mortality rate for infected healthy people under 60 is $0.1 \%$. This would mean that wearing facemasks would reduce one's risk of infection as a healthy person under 60 from $5 \%$ to $4.75 \%$; the risk of death from COVID-19 would drop from $0.005 \%$ to $0.00475 \%$ due to wearing facemasks. This does not seem like a sufficient risk reduction to justify mandatory facemasks in public.

Wearing masks is not terribly inconvenient, but it does have costs in terms of dollars, an energy burden from manufacturing and distribution, and pollution pressure on landfills, bodies of water and the environment in general. Given these considerations, and the evidence from RTCs, it would seem that mandates for wearing facemasks in public should be, at the least, reduced to recommendations, until there is definitive evidence that they are effective. For any other topic in medicine, one would expect a consensus to exist if all the available RTCs and meta-analyses found that a given intervention has no effect on the target problem. One would expect authorities to state that there is no need for the intervention. If the same standards were applied to public wearing of facemasks for COVID-19, there would be neither a mandate nor a recommendation for them. As discussed recently, facemask policies are only one of many problems with how medical authorities have handled the COVID-19 epidemic; many public health recommendations and statements have not been based on science [6].

\section{References}

1. Brainard JS, Jones N, Lake I, Hooper L, Hunter P (2020) Face masks and similar barriers to prevent respiratory illness such as COVID-19: A rapid systematic review. Medrxiv doi:10.1101/2020.04.01.20049528.

2. Cowling BJ, Zhou Y, Ip DK, Leung GM, Aiello AE (2010) Face masks to prevent transmission of influenza virus: a systematic review. Epidemiology of Infections 138: 449-456. [crossref] 
3. Xiao J, Shiv EYC, Gao H, Wong JY, Fong MW, et al. (2020) Nonpharmaceutical measures for pandemic influenza in non-healthcare settings - personal protective and environmental measures. Emerging Infectious Diseases 26: 967-975.

4. Aggarwhal N, Dwarakananthan V, Gautham N, Ray A (2020) Facemasks for prevention of viral respiratory infections in community settings: A systematic review and meta-analysis. Indian Journal of Public Health 64: 192-200.
5. Greenhalgh T, Schmid MB, Czypionka T, Bassler D, Gruer L (2020) Facemasks for the public during the COVID-19 crisis. British Medical Journal 369: m1435. [crossref]

6. Ross CA (2020) Thoughts on COVID-19. Journal of Neurology and Neurocritical Care 3: 1-3. 